\title{
The application of gray theory on wire cut electric discharge machine non-resistance sparkle circuit prediction
}

\author{
Shao-Hsien Chen ${ }^{1} \cdot$ Chen-Peng Wang ${ }^{2}$ \\ Received: 8 July 2017 / Accepted: 2 November 2017 / Published online: 30 November 2017 \\ (C) The Author(s) 2017. This article is an open access publication
}

\begin{abstract}
The development and research of the green manufacturing technology is relatively important. In order to improve processing efficiency of modern wire cut electric discharge machine (WEDM) and energy saving, a large number of researches and developments of the discharge circuit that is the major and most important technology of wire cutting machine are conducted. Currently, in the most discharge circuits of WEDM adopt the resistor for current-limiting which is the energy block when the resistor is used to turn off the discharge current, resulting in a lot of energies that are converted into heat energy from electrical energy, which are mainly generated by resistor. However, these heat energies will cause the increase on temperature in the discharge circuit, which affects the processing efficiency and energy. Thus, some cooling devices should be arranged around the discharge circuit in order to reduce the temperature. This study adopts the WEDM as laboratory equipment to design and improve the discharge circuit, uses the DC-DC and the electronic voltage regulation technology to convert the original energy bled by the resistor directly into the energy required in machining, and then applies the design of non-resistance sparkle circuit to realize the energy-saving effect. The experiment testing the power consumption differences of WEDM shows that the energy can be saved by 10 to $15 \%$ performance.
\end{abstract}

Keywords Wire cut electric discharge machine $($ WEDM) $\cdot$ Non-resistance sparkle circuit $\cdot$ Machine tools

\section{Introduction}

This study conducts the research and design on the discharge circuit of wire cut electric discharge machine (WEDM) system so that the original resistance discharge circuit is designed and improved to be non-resistance discharge circuit. And the purpose thereof is to use the concept of energy regeneration to convert the original energy bled into resistor to feed back to discharge energy, and then, the device of power can directly reduce electricity consumption, so as to realize that the green machine tool technology is applied to wire cutting machine.

The wire cutting machine tool is one of the main tools for precision mold processing, and many mold products can obtain related artifacts only through the machining of WEDM,

Shao-Hsien Chen

e6036@ncut.edu.tw

1 Department of Mechanical Engineering, National Chin-Yi University of Technology, Taichung City, Taiwan

2 Department of R\&D, Ching Hung Machinery \& Electric Industrial CO., LTD, Taichung, Taiwan which also can be said to be an extremely important step in industrial manufacture. Moreover, currently, with the global warming, the consciousness of environmental protection gradually came up, which makes the green processing technology more important. However, the green machine tool in which Lee [1] mentioned described that only $25 \%$ of consumed energy was used for machining and the rest was used for transmission loss or the energy of standby energy. Therefore, the renewable materials are used to make the machine tools reduce the size and weight so that the consume materials can be recycled and regenerated.

The modern machine tools are designed based on considering the goals of energy efficiency and green manufacture, DMG [2] has proposed to reduce at least $75 \%$ of energy in non-productive time by the use of energy save, whose actuation principle is to shift the machine tool to power-down mode in non-productive time, so as to reduce the energy use of peripheral devices, such as the high-efficiency motors with optimum speed to match the torque; its purpose is to make the energy with more efficient application.

WEDM is common processing equipment for current mold processing, and the processing molds are the parts requiring 
straightness and roundness, such as the punch and guide post of stamping die. However, the plastic mold can obtain the surface gloss of finished products by using the intensive loop of WEDM, and the taper machining of WEDM can facilitate the mold release of plastic mold. The WEDM also has advantages in parts processing. As for the small and parts without burrs, such as the parts used for watch, or medical equipment parts requiring good processing environment, the WEDM has a very good play space. This study proposes the concept of renewable energy for the discharge circuit of WEDM, which converts the original energy bled into resistor to feed back to discharge energy so that the customers can directly reduce electricity consumption, so as to realize the instance of applying the green technology to WEDM. The main application is to apply multiple transistors to control the discharge, so as to recycle the energy by the transistors when the discharge energy is turned off.

\section{Description of WEDM discharge principle}

\subsection{Description of non-resistance sparkle circuit}

The wire cutting machine tool contains the motion control system and discharge circuit system, as described below. Motion control system: Motion control system which cooperates with discharge module to process is the bed mechanism of drive machine tool. Three major control loops of servo control system are respectively [3] (1) speed control circuits, (2) position control circuit, and (3) current control circuit. The control of servo system control is usually composed of the instruction system, control system, drive system, and mechanical system.[4]

Power control system: The power control system is the important core technology of various discharge machines, which leads various important indicators, such as the discharge efficiency, surface roughness in discharge, and so on, while the current power control system is developed according to the principle mentioned in of discharge principle and characteristics [5-7].

Therefore, the metal removal rate refers to the processing capacity to the material being processed during the machining of wire cut discharge, which is related to the power inside of the power system of WEDM.

The consumed power of discharge machining $(W)$

$W=V \times I \times T \times F_{q}$

where $V$ is the discharge voltage (Volt), $I$ discharge current (Amp), $T$ current duration (s), and $F_{\mathrm{q}}$ discharge frequency $(\mathrm{KHz})$.
Removing the energy $(E)$ required by a unit volume of material, wherein the denominator is the metal removal rate

$E=\frac{W}{G \times H \times F d}$

where $G$ is the machining width (mm), $H$ workpiece thickness $(\mathrm{mm})$, and $F_{d}$ feed rate $(\mathrm{mm} / \mathrm{s})$.

As for the non-resistance sparkle circuit, the energy of counter electromotive force, which is originally bled by resistor during the discharge process, can improve the heat generated through energy regeneration loop. The main reason is that the new loop no longer needs the resistor, which is known as the non-resistance sparkle circuit, and its working block diagrams are shown in Fig. 1.

When the non-resistance sparkle circuit is used for machining, it reuses the energy originally bled; thus, it is known as energy-saving circuit, which is to overplay the energy bled by processing resistor with the original energy by conversion, so as to increase the original discharge energy and avoid considering the problem of cooling due to the heat energy produced by resistor $[8,9]$.

As for the corresponding relationship and operating principle of non-resistance sparkle circuit and discharge circuit, Fig. 1 shows a non-resistance sparkle circuit. The external main supply AC $220 \mathrm{~V}$ is supplied to the controller and transformer, and the controller is mainly responsible for overall control. This block diagram only illustrates the part of discharge control. The controller of the discharge circuit controls the discharge timing sequence, which is mainly processing conditions such as ON time and OFF time. The main supply is transformed to be DC power supply after entering in transformer and then will be converted into required discharge voltage value through discharge power. It is mainly provided for discharge power and mainly used as the source of discharge energy. This energy will be stored in capacitor $\mathrm{C} 1$ firstly, waiting for on and off of the discharge switch which is controlled by discharge control.[4, 10]

In machining, the controller will control the function of discharge control according to the set machining parameters. The controller will drive the discharge switch to conduct action of ON and OFF in discharge when it confirms that the environment has reached the discharge condition, which can use the time of ON and OFF to compose the discharge and stop time of the set value of discharge condition. For the action of switching ON or OFF, it will produce counter electromotive force resulted in the quick open and close of the discharge switch. For a conventional circuit, this back EMF is discharged by resistance. However, in a non-resistance circuit, this energy is stored in the capacitor $\mathrm{C} 2$ directly and then boosted by the recycle circuit. When the voltage in the recycle circuit is higher than that in the $\mathrm{C} 1$, it will automatically flow to $\mathrm{C} 1$ as the energy used 
Fig. 1 Working block diagram of non-resistance sparkle circuit

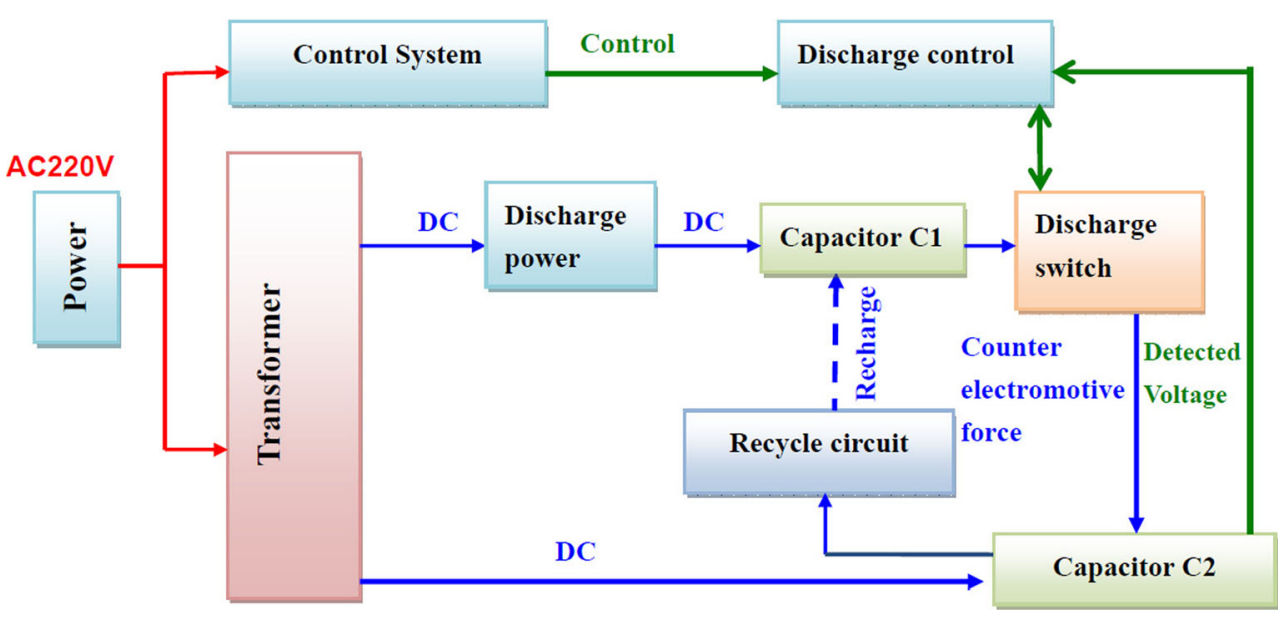

for next discharge. Therefore, such loop consists of the basic operation of non-resistance sparkle circuit.

\subsection{Internal architecture description of non-resistance sparkle circuit}

This section illustrates the internal structures for resistance sparkle circuit structure diagram and non-resistance sparkle circuit structure diagram, wherein the resistance sparkle circuit structure diagram is shown in Fig. 2, the left block is LV (low voltage) provided by striking voltage, which switches through Q2. The intermediate block of HV is a high voltage energy, which discharges the energy through Q1, enabling the energy to be transmitted between $\mathrm{D}$ and $\mathrm{W}$ for discharge. The right block is functioned as a resister used for bleeding. The counter electromotive force of Q1 produced when opening and closing the energy is absorbed into capacitor $\mathrm{C} 2$, which is detected by the CMP1. When the $\mathrm{C} 2$ is close to saturation, the energy will flow to R2 for bleeding by Q3, which will produce heat through the resister, namely the electrical energy is converted into heat.

Non-resistance sparkle circuit architecture graph is shown in Fig. 3, the counter electromotive force generated by Q1 stores the energy in the capacitor $\mathrm{C} 2$, and CMP1 detection circuit is responsible for detecting the energy of capacitor C2. The boost DC-to-DC converter composed by Q3, Q4, Q5, Q6, and T1 will be enabled when the storage is close to saturation. The voltage will be boosted, and the voltage is rectified through the full bridge rectifier D2 and finally flows into $\mathrm{C} 1$ used for energy storage in discharge as discharge energy.

The actual circuit board diagram of non-resistance sparkle circuit will be mounted on WEDM actually to conduct discharge test to verify the effect of nonresistance sparkle circuit. The block diagram in Fig. 4 describes the discharge power; when the power supply device generates DC power, the switch will conduct the action of ON and OFF. When the switch is turned on, it will produce discharge power through discharge device for discharge. However, when discharge is stopped, it needs to transfer the residual energy to bleed-resistor for bleeding. Therefore, based on the above actions, the formula of discharge power can be as follows:

$$
W=W_{\mathrm{d}}-W_{\mathrm{h}}
$$

where $W$ is the discharge power, $W_{\mathrm{d}} \mathrm{DC}$ power, and $W_{\mathrm{h}}$ heat consumption
Fig. 2 Resistance discharge circuit architecture diagram

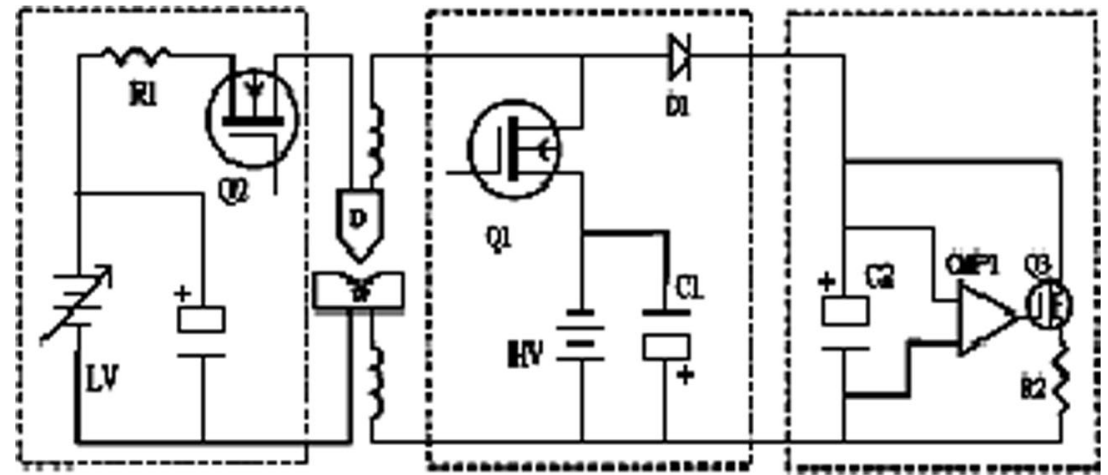


Fig. 3 Non-resistance discharge circuit architecture diagram

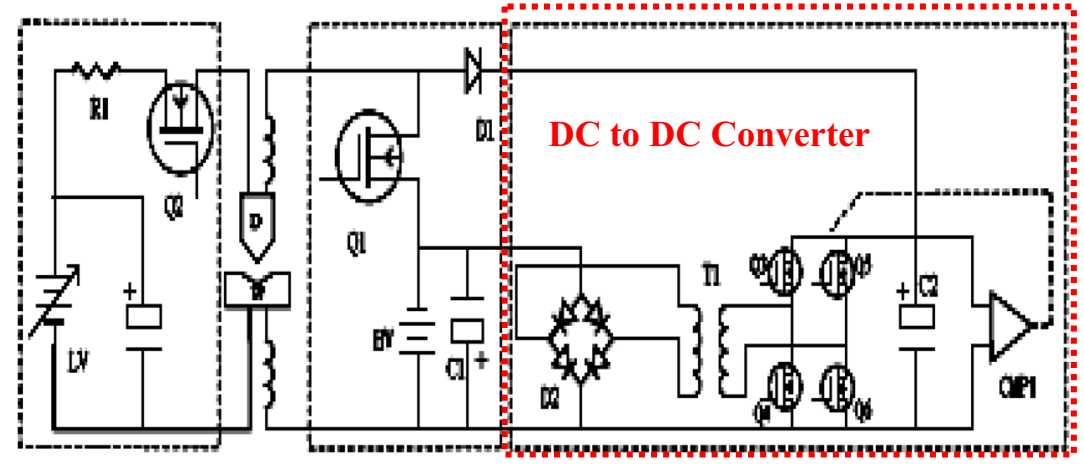

\section{Construction of prediction model}

\subsection{Gray prediction GM $(1,1)$ model}

The gray prediction model is primary the $\operatorname{GM}(1,1)$ developed by Deng [11-13], mainly applied under conditions of less model information, more impact variables, and uncertain behavior models, and the operation model includes the works of correlation analysis, model construction, prediction and control, and so on. [3]

The GM $(n, h)$ as a GM is a dynamic model where $n$ is the order of the differential function and $h$ is the number of variables in the differential function. Thus, GM $(1,1)$ is a firstorder differential function and is a method based on training values for prediction [14-16]. It features prediction analysis with less training values. The prediction process of the GM (1, 1) model at present is shown below:

Step 1: Primitive sequence and experimental values

$$
x^{(0)}=\left(x^{(0)}(1), x^{(0)}(2), x^{(0)}(3), \ldots \ldots, x^{(0)}(n)\right), n \geq 2
$$

Step 2: Conduct the accumulated generating operation (AGO)

Let $x^{(1)}$ be the transformation sequence of $x^{(0)}$ at $\mathrm{T}$

$x^{(1)}=\left(x^{(1)}(1), x^{(1)}(2), x^{(1)}(3), \ldots \ldots \ldots . ., x^{(1)}(n)\right)$

$x^{(1)}(k)=\sum_{m=1}^{k} x^{(0)}(m)$

Fig. 4 Discharge power block diagram
Consequently, model of the first-order differential eq. $\mathrm{GM}(1,1)$ is:

$\frac{d x^{(1)}}{d t}+a x^{(1)}=b$

wherein, $t$ is the independent variable, $a$ the development coefficient, and $b$ the gray control variable.

Step 3: Conversion treatment of the former and latter terms $\frac{d x^{(1)}}{d t} \rightarrow x^{(1)}(k+1)-x^{(1)}(k)$

Through Inverse Accumulated Generating Operation (IAGO), it can be learned that:

$x^{(1)}(k+1)-x^{(1)}(k)=x^{(0)}(k+1)$

If $\alpha=0.5$, its definition in $x_{1}^{(1)}(t)$ would be:

$x^{(1)}(k) \rightarrow 0.5 x^{(1)}(k)+0.5 x^{(1)}(k-1)=z^{(1)}(k)$

After collation, it is known:

$\frac{d x^{(1)}}{d t}+a x^{(1)}=b \rightarrow x^{(0)}(k)+a z^{(1)}(k)=b$

Step 4: Determine $a, b$ by least square method.

$p=\left[\begin{array}{l}a \\ b\end{array}\right]=\left(B^{T} B\right)^{-1} B^{T} Y_{n}$

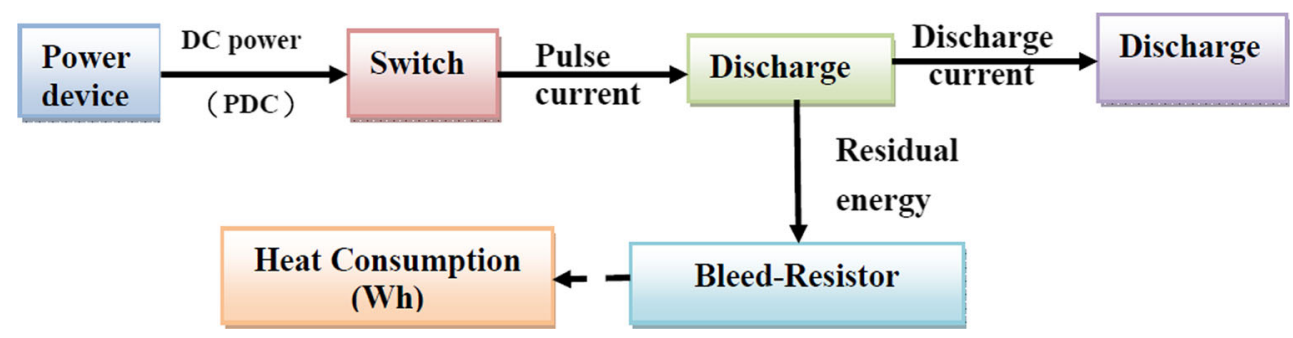


In which, the accumulated matrix $B$ and constant term $Y_{n}$ are respectively:

$B=\left[\begin{array}{cc}-z^{(1)}(2) & 1 \\ -z^{(1)}(3) & 1 \\ \vdots & 1 \\ -z^{(1)}(n) & 1\end{array}\right] \quad Y_{n}=\left[\begin{array}{c}x(2) \\ x(3) \\ \vdots \\ x(n)\end{array}\right]$

Step 5: Construct the gray prediction model

The equation in $\operatorname{GM}(1,1)$ is of:

$\frac{d x^{(1)}}{d t}+a x^{(1)}=b$

Wherein, the primitive condition of $x^{(1)}$ is $x^{(0)}(1)=x^{(1)}(1)$.

According to the calculation method of ordinary differential equations (ODE), it can obtain the discretization response equation:

$\hat{x}^{(1)}(k+1)=\left(x^{(0)}(1)-\frac{b}{a}\right) e^{-a(k-1)}+\frac{b}{a}, k \geq R$

$\hat{x}^{(1)}(k+1)=\left(x^{(0)}(1)\right) e^{-a k}+\frac{b}{a}\left(1-e^{-a k}\right), k \geq 0$

Step 6: Process with LAGO

$\hat{x}^{(0)}(k+1)=\left(1-e^{a}\right)\left(x^{(0)}(1)-\frac{b}{a}\right) e^{-a k}$

\subsection{Improved GM $(1,1)$ model}

Generally, the equations in $\operatorname{GM}(1,1)$ are the first-order differential equations, which all belong to convergence type, while the experimental value in this study is of progressive increase pattern which is of divergence type; thus, it is modified into divergence form in the first-order differential equations and results in accumulated errors in IAGO. As a result, it is necessary to be converted and changed in the whitening formula. The following steps can be obtained after being modified: [17, 18]

Step 1: Primitive sequence and experimental values

$x^{(0)}=\left(x^{(0)}(1), x^{(0)}(2), x^{(0)}(3), \ldots \ldots, x^{(0)}(n)\right), n \geq 2$

Step 2: Execute the accumulated generating operation (AGO)

Let $x^{(1)}$ be the transformation sequence of $x^{(0)}$ at $T$

$x^{(1)}=\left(x^{(1)}(1), x^{(1)}(2), x^{(1)}(3), \ldots \ldots \ldots, x^{(1)}(n)\right)$

$x^{(1)}(k)=\sum_{m=1}^{k} x^{(0)}(m)$
Consequently, model of the first-order differential equation GM $(1,1)$ after modification is:

$\frac{d x^{(1)}}{d t}-a x^{(1)}=b$

wherein, $t$ is the independent variable, $a$ the development coefficient, and $b$ the gray control variable.

Step 3: Conversion treatment of the former and latter terms

$\frac{d x^{(1)}}{d t} \rightarrow x^{(1)}(k+1)-x^{(1)}(k)$

Through inverse accumulated generating operation (IAGO), it can be learned that:

$x^{(1)}(k+1)-x^{(1)}(k)=x^{(0)}(k+1)$

If $\alpha=0.5$, its definition in $x_{1}^{(1)}(t)$ would be:

$x^{(1)}(k) \rightarrow 0.5 x^{(1)}(k)+0.5 x^{(1)}(k-1)=z^{(1)}(k)$

After collation, it is known:

$\frac{d x^{(1)}}{d t}-a x^{(1)}=b \rightarrow x^{(0)}(k)-a z^{(1)}(k)=b$

Step 4: Determine $a, b$ by least square method.

$p=\left[\begin{array}{l}a \\ b\end{array}\right]=\left(B^{T} B\right)^{-1} B^{T} Y_{n}$

In which, the accumulated matrix $B$ and constant term $Y_{n}$ are respectively:

$B=\left[\begin{array}{cc}-z^{(1)}(2) & 1 \\ -z^{(1)}(3) & 1 \\ \vdots & 1 \\ -z^{(1)}(n) & 1\end{array}\right] \quad Y_{n}=\left[\begin{array}{c}x(2) \\ x(3) \\ \vdots \\ x(n)\end{array}\right]$

Step 5: Construct the gray prediction model

Its equation in $\operatorname{GM}(1,1)$ is of:

$\frac{d x^{(1)}}{d t}-a x^{(1)}=b$

wherein, the primitive condition of $x^{(1)}$ is $x^{(0)}(1)=x^{(1)}(1)$. 
According to the solutions to ordinary differential equations (ODE), it can obtain the discretization response equation:

$\hat{x}^{(1)}(k+1)=\left(x^{(0)}(1)+\frac{b}{a}\right) e^{a(k-1)}-\frac{b}{a}, k \geq N$

Step 6: Process with LAGO

$\hat{x}^{(0)}(k+1)=\left(1-e^{-a}\right)\left(x^{(0)}(1)+\frac{b}{a}\right) e^{a(k-1)}$

Step 7: Corrected value $u$ after improvement

It mainly improves the error between the first point $x^{(0)}(1)$ of the primitive data and the first point $\hat{x}^{(0)}(1)$ of the prediction value [16]:

$u=\frac{b}{a}-\left(x^{(0)}(1)+\frac{b}{a}\right) e^{-a}$

The error amount $u(k)$ of the rest points is determined as:

$u(k)=e^{a(k-1)}\left(x^{(0)}(1)+u\right)-x^{(0)}(k)$

Step 8: Training value after modification

Take $u(k)$ as the primitive data to get $u(k+1)$ by exponential smoothing, and its corrected value is $\bar{x}^{(0)}(k)$, shown as:

$\bar{x}^{(0)}(k)=\hat{x}^{(0)}(k)-u(k)$

\section{Experimental planning and equipment}

This study analyzes and tests a resistive circuit and nonresistance sparkle circuit. The first stage sets the machining parameters and analyzes the impacts of different discharge circuits on machining energy, so as to verify the features of energy-saving of non-resistance sparkle circuit on discharge machining; the second stage is to set machining parameters and analyzes the impacts of different discharge circuits on machining speed; the third stage changes the machining parameters and analyzes the impacts of different discharge circuits on machining speed.

This experiment adopts the WEDM produced by CHMER Group as experimental equipment, with the main device including the mechanical structure composed by servo control of five axes of $X, Y, U, V$, and $Z$, as well as feed device driven by cooper wire, machining power supply systems, CNC

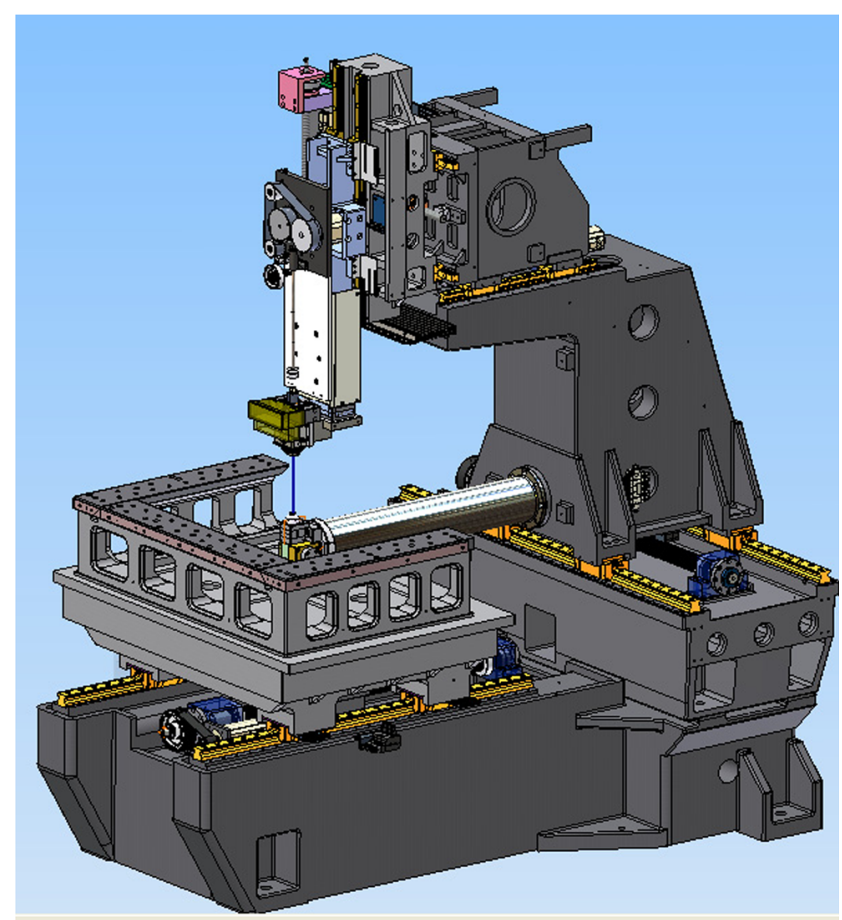

Fig. 5 Structure of WEDM

controller, and machining liquid supply device. Equipment thereof is shown in Fig. 5, and the mechanical specifications are listed in Table 1 [19-21].

\section{Results and discussion}

\subsection{Impact on the machining power from different circuits}

This study compares the power consumption of machining between non-resistance sparking circuit and resistance sparking circuit. The machining parameters are set as $\mathrm{ON}=$ $7, \mathrm{OFF}=8, \mathrm{AN}=5, \mathrm{AFF}=9, \mathrm{SV}=38, \mathrm{FR}=15, \mathrm{WF}=7, \mathrm{WT}$

Table 1 Table of specifications and performance

\begin{tabular}{lll}
\hline Model & Unit & G32S Type \\
\hline$X, Y$ axis & $\mathrm{mm}$ & $360 \times 250$ \\
$U, V, Z$ axis & $\mathrm{mm}$ & $60 \times 60 \times 220$ \\
Max workpiece $(W \times D \times H)$ & $\mathrm{mm}$ & $725 \times 560 \times 215$ \\
Weight of max workpiece & $\mathrm{kg}$ & 300 \\
Motor system & $\mathrm{axis}$ & $\mathrm{AC}$ servo motor \\
Wire diameter (standard) & $\mathrm{mm}$ & $\mathbb{C} 0.15 \sim C 0.3(\mathbb{C} 0.25)$ \\
Wire tension & $\mathrm{gf}$ & $300 \sim 2500$ \\
N.W(including power and & $\mathrm{kg}$ & 2375 \\
$\quad$ water system) & $\mathrm{mm}{ }^{2} / \mathrm{min}$ & $150 \sim 200$ \\
Machining speed & $\mathrm{Ra}$ & $0.5 \sim 0.6$ (three-pole processing) \\
Surface granularity &
\end{tabular}




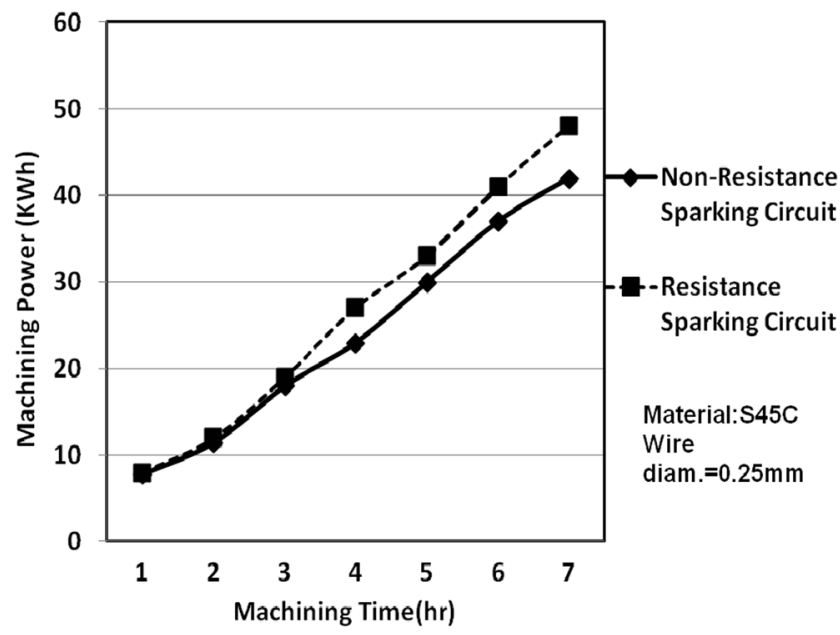

Fig. 6 Power consumption comparison between non-resistance circuit and resistance circuit

$=8$, and $\mathrm{WL}=6$. An ammeter is used to observe the machining change of the external input power, with measurement results shown in Fig. 6. If the machining time is to $1 \mathrm{~h}$, the impact is not significant. After machining for $7 \mathrm{~h}$, the impacts of non-resistance sparking circuit and resistance sparking become more significant. In the case that the cutting time is $T=$ $1 \mathrm{~h}$, the difference of cutting power is $0.2 \mathrm{KWh}$. When the cutting time is increased to $T=7 \mathrm{~h}$, the difference of cutting power is $6 \mathrm{KWh}$. The main reason is that the energy discharged through the resistor in machining, after conversion and loopback, returns to the workpiece and the electrode, which is recycled and reused. In this way, it reduces the power consumption and temperature generation in machining circuit.

\subsection{Analysis and comparison on the application of gray prediction between different machining time}

The longer the cutting time, the greater the power consumption of machining. From Eqs. 1 and 2, the power

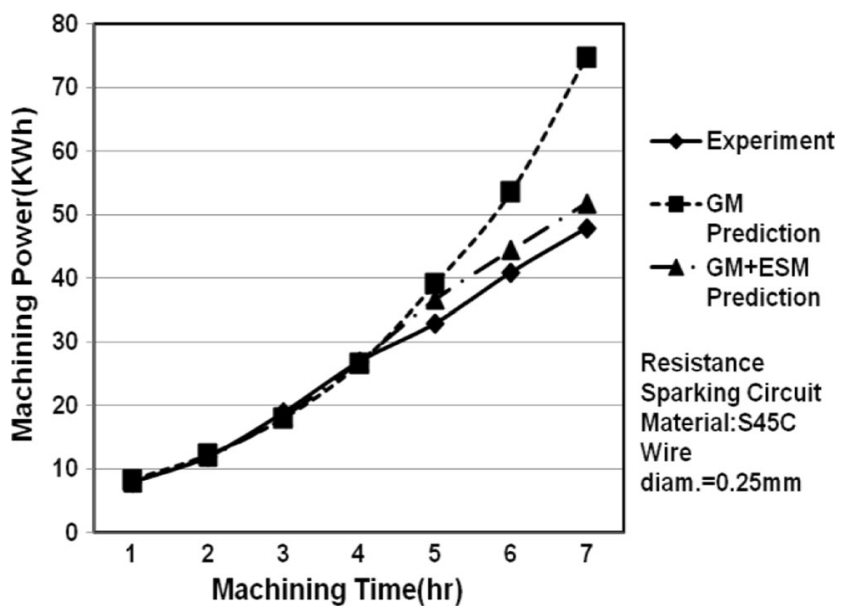

Fig. 7 Comparison of machining time and power consumption between different prediction models
Table 2 Comparison between original gray prediction model and improved gray prediction model

\begin{tabular}{llllll}
\hline \multicolumn{6}{l}{ Resistance sparking circuit } \\
\hline Time & Test & GM $(1,1)$ & Error & GM $(1,1)+$ ESM & Error \\
\hline 1 & 8 & 8.34 & 0.34 & 8 & 0 \\
2 & 12 & 12.27 & 0.27 & 11.96 & 0.03 \\
3 & 19 & 18.07 & 0.92 & 18.32 & 0.67 \\
4 & 27 & 26.59 & 0.40 & 27.00 & 0.002 \\
5 & 33 & 39.13 & 6.13 & 36.70 & 3.70 \\
6 & 41 & 53.59 & 12.5 & 44.45 & 3.45 \\
7 & 48 & 74.76 & 26.76 & 51.88 & 3.88 \\
MAPE & & & 3.87 & & 0.55 \\
RMSE & & & 11.42 & & 2.42 \\
\hline
\end{tabular}

consumption of 7-h machining for general sparking circuit is about $50 \mathrm{KWh}$. In the gray prediction model and improved gray prediction GM $(1,1)$ model, the error becomes significantly great after 4-h machining, among which the result of the improved gray model is close to actual measurement value, as shown in Fig. 7 Therefore, the improved gray prediction GM $(1,1)+$ ESM is better than general gray prediction GM $(1,1)$.

The comparison of gray prediction and improved gray prediction models is shown in Table 2, from which it can be known that as for mean absolute percentage error (MAPE), the error after improvement 0.55 is smaller than 3.87 ; as for root-mean-square error (RMSE), the error after improvement 2.42 is smaller than 11.42 . Therefore, when the values of the original experiment are incremented, the improved gray prediction model GM $(1,1)+$ ESM can be used to reduce the error.

When the cutting time becomes longer, the machining power consumption will significantly increase. When the

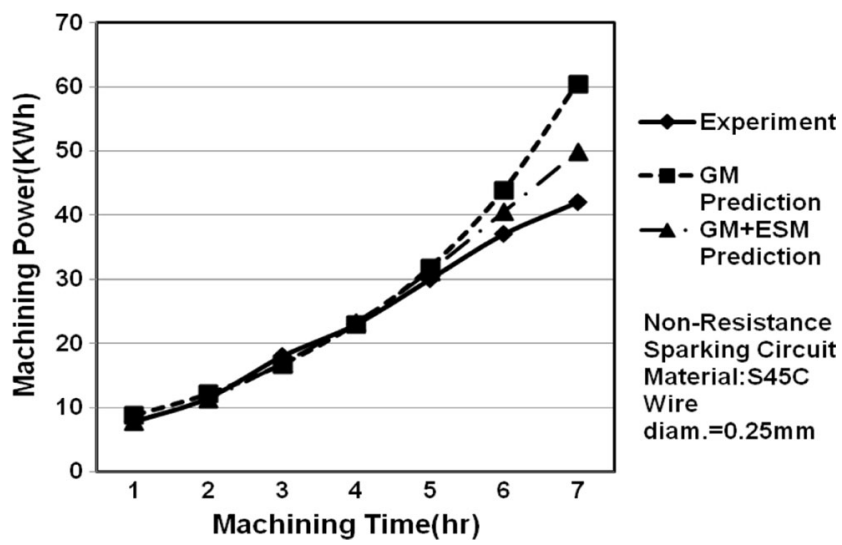

Fig. 8 Comparison of machining time and power consumption between different prediction models 
Table 3 Comparison between original gray prediction model and improved gray prediction model

Non-resistance sparking circuit

\begin{tabular}{llllll}
\hline Time & Test & GM $(1,1)$ & Error & GM $(1,1)+$ ESM & Error \\
\hline 1 & 7.8 & 8.81 & 1.01 & 7.8 & 0 \\
2 & 11.5 & 12.14 & 0.64 & 11.30 & 0.19 \\
3 & 18 & 16.74 & 1.25 & 16.86 & 1.13 \\
4 & 23 & 23.09 & 0.09 & 23.28 & 0.28 \\
5 & 30 & 31.83 & 1.83 & 31.27 & 1.27 \\
6 & 37 & 43.88 & 6.88 & 40.51 & 3.51 \\
7 & 42 & 60.50 & 18.50 & 49.92 & 7.92 \\
MAPE & & & 2.78 & & 1.13 \\
RMSE & & & 7.52 & & 3.34 \\
\hline
\end{tabular}

non-resistance sparking circuit is used, the power consumption of 7-h machining is about $40 \mathrm{KWh}$, which saves the power consumption by $10 \sim 20 \%$ when compared to general sparking circuit. In the gray prediction and improved gray prediction model $\operatorname{GM}(1,1)$, the error significantly increases after $5 \mathrm{~h}$. The result of the improved gray prediction model is close to the actual measurement value, as shown in Fig. 8 Therefore, the improved gray prediction model GM $(1,1)+$ ESM is better than the general gray prediction $\operatorname{GM}(1,1)$.

The comparison of gray prediction and improved gray prediction models is shown in Table 3, from which it can be known that as for MAPE, the error after improvement 1.13 is smaller than 2.78; as for RMSE, the error after improvement 3.34 is smaller than 7.52. Therefore, when the values of the original experiment are incremented, the improved gray prediction model GM $(1,1)+$ ESM can be used to reduce the error.

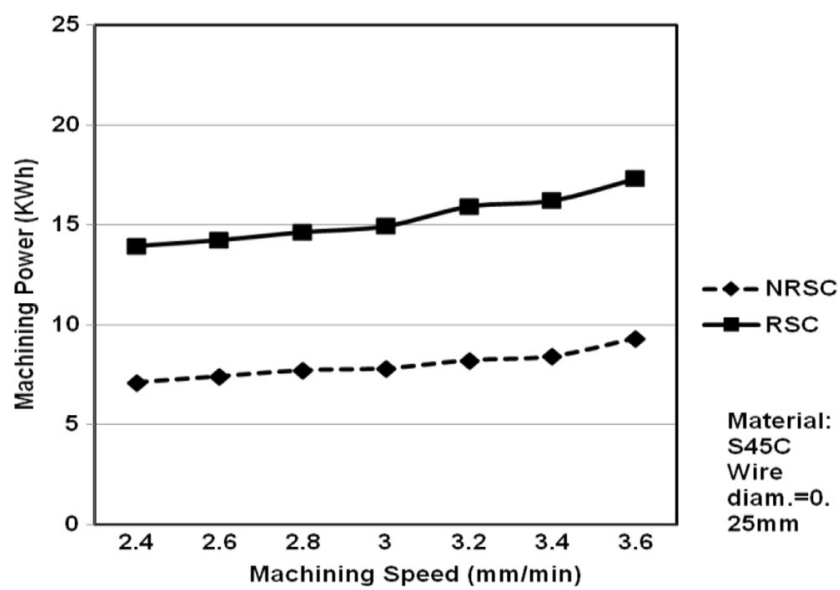

Fig. 9 Power consumption comparison of different machining velocities between non-resistance and resistance circuits

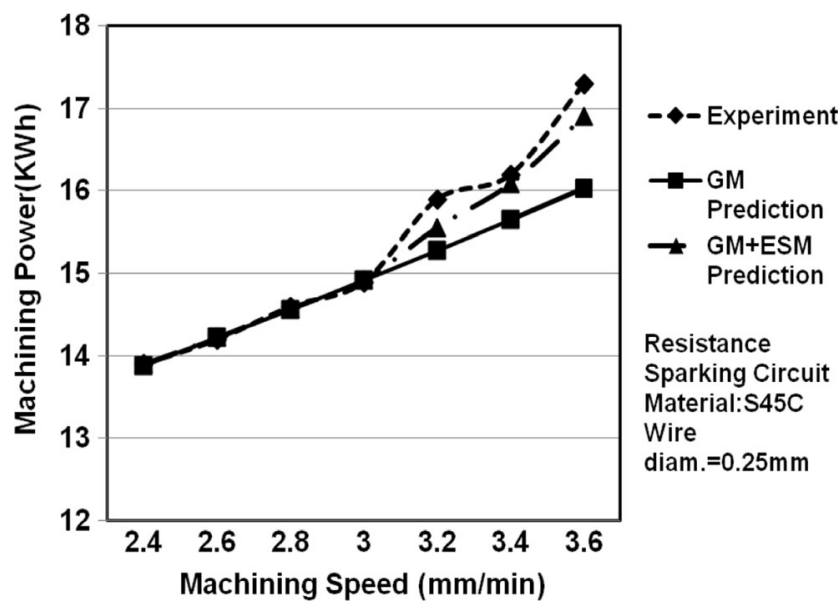

Fig. 10 Comparison of machining velocities and power consumption between different prediction models

\subsection{Comparison of power consumption under the same velocity}

WEDM's cutting power consumption is mainly affected by parameters of the sparking voltage, sparking current, duration of the current, sparking frequency, material properties, and cutting velocity which are correlated. From the Eqs. 1 and 2, the impact from resistance circuit and non-resistance circuit on power consumption is observed when the cutting velocity is changed. At the cutting velocity of $V=2.4$, the difference of the cutting power is $6.8 \mathrm{KWh}$; and when the cutting velocity is increased to $V=3.6$, the difference in cutting power is $8 \mathrm{KWh}$, as shown in Fig. 9. When the machining velocity is same, the cutting power consumption of the non-resistance sparking circuit is less than that of the resistance sparking circuit, mainly because the heat consumption power causes the reduction of the sparking power. From Eq. 3, it can be known that in the increase of cutting velocity, the cutting power is consumed more significantly.

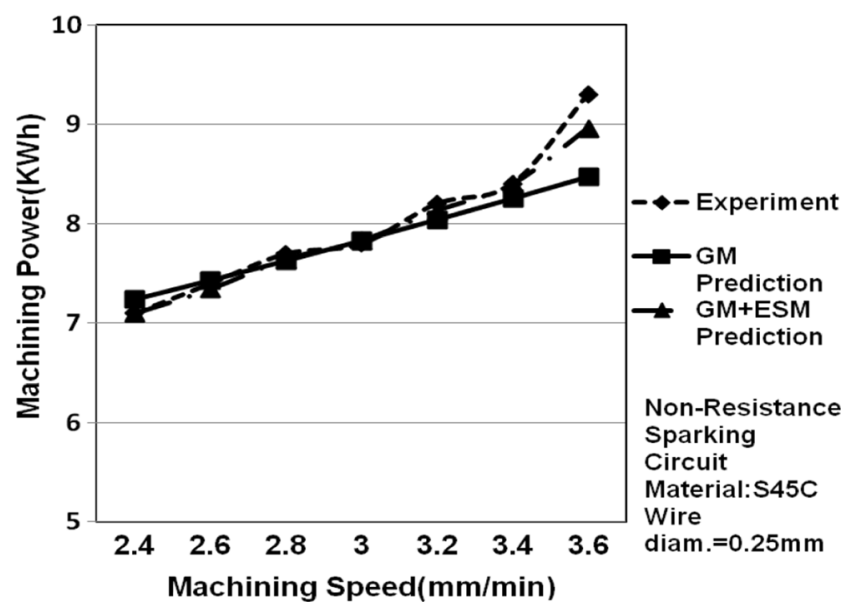

Fig. 11 Comparison of machining velocities and power consumption between different prediction models 
Table 4 Comparison between the original gray prediction model and the improved gray prediction model

\begin{tabular}{llllll}
\hline \multicolumn{2}{l}{ Resistance sparking circuit } & \multicolumn{1}{l}{} \\
\hline Machining velocity & Test & GM $(1,1)$ & Error & GM $(1,1)+$ ESM & Error \\
\hline 2.4 & 13.9 & 13.88 & 0.02 & 13.9 & 0 \\
2.6 & 14.2 & 14.22 & 0.02 & 14.222 & 0.022 \\
2.8 & 14.6 & 14.56 & 0.04 & 14.575 & 0.025 \\
3 & 14.9 & 14.92 & 0.02 & 14.9197 & 0.0197 \\
3.2 & 15.9 & 15.28 & 0.62 & 15.557 & 0.343 \\
3.4 & 16.2 & 15.65 & 0.55 & 16.089 & 0.111 \\
3.6 & 17.3 & 16.03 & 1.27 & 16.903 & 0.397 \\
MAPE & & & 0.184 & & 0.056 \\
RMSE & & & 0.573 & & 0.203 \\
\hline
\end{tabular}

\subsection{Analysis and comparison on the application of gray prediction between different machining velocities}

When the cutting velocity increases, the machining power consumption will significantly increase, as shown in Figs. 10 and 11. In the gray prediction model and improved gray prediction model GM $(1,1)$, the result of the improved gray prediction model is close to the actual measurement value. Therefore, the improved gray prediction model GM $(1,1)+$ ESM is better than the general gray prediction GM $(1,1)$.

For the relationship between the cutting velocity and the machining energy, as shown in Table 4, comparison between the original gray prediction model and the improved gray prediction model and the general sparking circuit comparison table, as for MAPE, the error after improvement 0.056 is smaller than 0.184; as for RMSE, the error after improvement 0.203 is smaller than 0.573 . Therefore, when the values of the original experiment are incremented, the improved gray prediction model GM $(1,1)+$ ESM can be used to reduce the error. As for the non-resistance sparking circuit, as shown in Table 5, as for MAPE, the error after improvement 0.048 is smaller than 0.098; as for RMSE, the error after improvement 0.135 is smaller than 0.329 .

\section{Conclusion}

This study mainly makes use of the back electromotive force discharged by the resistor in the machining process of EDM, which is recycled to the capacitor, then boosted and recharged to the energy originally used for sparking. In this way, it could eliminate the original resistor, which makes the heat converted from electric energy in the resistor no longer exist. On the other hand, the recycled energy has the energysaving effect, which realizes the recycle and re-use of the energy in machining. The following are the focuses sorted from the experiment.

A. The circuit design that the original resistance circuit is stored and boosted back to the charging and discharging energies has the energy-saving effect; as the resistor is removed, the heat generated by the resistor is also removed, which realizes the purpose of green manufacturing.
Table 5 Comparison table between original gray prediction model and improved gray prediction model

\begin{tabular}{|c|c|c|c|c|c|}
\hline \multicolumn{6}{|c|}{ Non-resistance sparking circuit } \\
\hline Machining velocity & Test & GM $(1,1)$ & Error & $\mathrm{GM}(1,1)+\mathrm{ESM}$ & Error \\
\hline 2.4 & 7.1 & 7.2433 & 0.1433 & 7.1 & 0 \\
\hline 2.6 & 7.4 & 7.4348 & 0.0348 & 7.3448 & 0.0552 \\
\hline 2.8 & 7.7 & 7.6314 & 0.0686 & 7.6452 & 0.0548 \\
\hline 3 & 7.8 & 7.8332 & 0.0332 & 7.8294 & 0.0294 \\
\hline 3.2 & 8.2 & 8.0404 & 0.1596 & 8.1284 & 0.0716 \\
\hline 3.4 & 8.4 & 8.253 & 0.147 & 8.3977 & -0.0023 \\
\hline 3.6 & 9.3 & 8.4712 & 0.8288 & 8.9593 & -0.3407 \\
\hline MAPE & & & 0.098 & & 0.048 \\
\hline RMSE & & & 0.329 & & 0.135 \\
\hline
\end{tabular}


B. Under the same machining velocity, as for the nonresistance circuit and the resistance circuit, the former can save the power by $10 \sim 15 \%$. Therefore, it can be said that the non-resistance circuit has energy-saving effect. When the cutting time is $T=1 \mathrm{~h}$, the difference of cutting power is $0.2 \mathrm{KWh}$; when the cutting time is increased to $T=7 \mathrm{~h}$, the difference of the cutting power is $6 \mathrm{KWh}$.

C. Under different velocities, the higher the cutting velocity is, the higher the machining energy is. The machining energy of the non-resistance sparking circuit is lower than that of general sparking circuit. As for the impact from resistance circuit and non-resistance circuit on cutting consumption power, when the cutting velocity is $V=$ 2.4 , the difference of the cutting power is $6.8 \mathrm{KWh}$; when the cutting velocity is increased to $V=3.6$, the difference of the cutting power is $8 \mathrm{KWh}$.

D. The factor of the least squares method causes too large error in the gray prediction model. After the exponential smoothing method is used for error correction, the error can be reduced, including the significant reduction of both MAPE and RMSE.

Open Access This article is distributed under the terms of the Creative Commons Attribution 4.0 International License (http:// creativecommons.org/licenses/by/4.0/), which permits unrestricted use, distribution, and reproduction in any medium, provided you give appropriate credit to the original author(s) and the source, provide a link to the Creative Commons license, and indicate if changes were made.

\section{References}

1. SODICK, AP250L, 2010, JIMTOF Booth Digest

2. Lee JY (2009) Green Machine Tool, Industrial Technology Research Institute

3. Deng JL (1989) Introduction of grey system theory. J Grey Syst $1(1): 1-24$

4. Iuliano L, Violante MG, Gatto A, Bassoli E (2008) Study of the EDM process effects on aluminum alloys. Int J Manuf Technol Manag 14(3/4):326-341
5. Wang W, Zhidong L, Wentai S, Yueqin Z, Zongjun T (2016) Surface burning of high-speed reciprocating wire electrical discharge machining under large cutting energy. Int J Adv Manuf Technol 87:2713-2720

6. Plaza S, Ortega N, Sanchez JA, Pombo I, Mendikute A (2009) Original models for the prediction of angular error in wire-EDM taper-cutting. Int J Adv Manuf Technol 44:529-538

7. Hsue WJ, Su H (2004) Removal analysis of WEDM's tapering process and its application to generation of precise conjugate surface. J Mater Process Technol 149:117-123

8. Chelladurai H, Jain VK, Vyas NS (2010) Development of a cutting tool condition monitoring system for high speed turning operation by vibration and strain analysis. Int J Adv Manuf Technol 37:471485

9. Hargrove SK, Ding D (2007) Determining cutting parameters in wire EDM based on workpiece surface temperature distribution. Int J Adv Manuf Technol 34(3-4):295-299

10. Tosun N, Cogun C, Pihtili H (2003) The effect of cutting parameters on wire crater sizes in wire EDM. Int J Adv Manuf Technol 21:857865

11. Deng J-1 (2000) Gray system theory and application. Gau Lih Book Co, Taipei

12. Deng J-1, Guo H (1996) Gray system theory and application. Chuan Hwa book co., LTD, Taipei

13. Hanxiong W, Deng J-1, Kunli W (1996) Introduction to gray analysis. Gau Lih Book Co, Taipei

14. Zhan Li M, Jin-Hua S (2011) Application of Grey-Markov model in forecasting fire accidents. Precede Engineering 11:314-318

15. Zhou P, Ang BW, Poh KL (2006) A trigonometric grey prediction approach to forecasting electricity demand. Energy Conver Manag 31:2839-2847

16. Lin CT, Chen LH (1999) Grey analysis of bank re-decreasing the required reserve ratio. J Grey Syst 4(2):119-132

17. Guozhen H, Guorong W (2008) Research on Prediction of Electricity Requirement of Modified GM $(1,1)$ Ash Forecasting Model. J Eng Sci Educ 5(3):446-458

18. Hung K-C, Hsu F-Y, Wu K-J, Wen K-L, Wu JH (2007) An enhanced $\operatorname{GM}(1,1)$ grey prediction approach with error term $\mu(\mathrm{k})$. J Grey Syst 10(2):59-68

19. Prihandana GS, Mahardika M, Hamdi M, Wong YS, Mitsui K (2009) Effect of micro-powder suspension and ultrasonic vibration of dielectric fluid in micro-EDM processes-Taguchi approach. Int J Mach Tools Manuf 49(12-13):1035-1041

20. Wang CP, Chen SH (2013) The study of equalization of performance for wire cut EDM. NCUT the Degree of Master, Taipei

21. Sinha SK (2010) Effects Of Wire Lag In Wire Electrical Discharge Machining (WEDM). Int J Eng Sci Technol 2(11):6622-6625 